\title{
Iris biometric recognition of latin american eyes in a white light controlled environment
}

\author{
Reconocimiento biométrico del iris en un ambiente controlado de luz blanca para \\ ojos latinoamericanos
}

\author{
D. A. Muñoz-Acosta iD ; D. R. Cardona-Cortes ; J. A. Cortés-Osorio iD
}

\begin{abstract}
Iris biometric recognition has taken a great interest in the last decade. Many private and public facilities need to improve their security access due to the increasing terrorist attacks, the growing number of fraud and identity theft among other felonies. The regular technologies are almost all based on infrared light illumination due to the ease of using this for texture approach recognition. Even Though this technique is suitable in most cases, the highest price of these cameras on the market demands the research of either daylight or a white light solution. There are some known iris databases such as CASIA and UBIRIS, but they are not from Latin-American eyes that have different occlusion and color issues. A white light controlled environment was used to take the set of pictures 500 that let us have a new color database to carry out the experiments based on the EigenIris technique. The best iris and pupil segmentations were obtained either on the red or green color layers of the RGB images at $100 \%$ of correct localization. In this research, a sensitivity of 0,82 and specificity of 0,85 was achieved with a modified version of the EigenIris method using the blue color layer.
\end{abstract}

Index Terms - biometrics, eigeniris, iris, latin american eyes, recognition, visible light.

Resumen - El reconocimiento biométrico del iris ha tomado un gran interés en la última década. Muchos establecimientos necesitan mejorar su acceso de seguridad debido al aumento de ataques terroristas, el número creciente de fraudes y robo de identidad, entre otros delitos. Las tecnologías regulares de biometría del ojo son basadas en la iluminación con luz infrarroja debido a la facilidad de uso de este método para el reconocimiento de la textura. A pesar de que esta técnica es adecuada en la mayoría de los casos, el precio más alto de dichas cámaras en el mercado exige la búsqueda de soluciones de luz del día o de luz blanca regular. Existen algunas bases de datos del iris conocidas como lo son CASIA y UBIRIS, pero no pertenecen a ojos de personas de América Latina, las cuales tienen diferentes características de oclusión y de color. Para esta investigación se usó un ambiente controlado de luz blanca que permitió tomar un conjunto de 500 imágenes que dieron origen a una nueva base de datos para llevar a cabo los experimentos basados en la técnica 2020 .

This manuscript was sent on August 31, 2019 and accepted on June 03,

D. A. Muñoz-Acosta is professor and researcher at the Universidad Tecnológica de Pereira, Carrera 27 \#10-02 Barrio Álamos - Risaralda Colombia, (alejandro2460@utp.edu.co).
EigenIris (Análisis de componentes principales con la modificación del clasificador, usando KNN y el clasificador Naive Bayes). Las mejores segmentaciones del iris y de la pupila se obtuvieron ya sea en la capa de color rojo o verde de la imagen RGB del ojo con un $100 \%$ de verdaderas localizaciones. En esta investigación se logró una sensibilidad de 0,82 y una especificidad de 0,85 en la identificación mediante el uso de la capa de color azul con una versión modificada propuesta del método del EigenIris y el clasificador Naive Bayes.

Palabras claves — biometría, eigenIris, iris, reconocimiento, ojos latinoamericanos, luz visible.

\section{INTRODUCTION}

INCE ancestral times, human beings have noticed the physical differences between them, which, in general, are unique and unrepeatable for each individual. In the measurements and parameters of the human body, these differences are called anthropometric characteristics and led to the development of techniques that use them for individual's recognition. Thanks to the vast complexity of the human body, a wide variety of patterns and differences have been found for the automatic recognition of people (biometrics), which has been applied mainly in security systems [1].

Thanks to their advantages and disadvantages, some biometric methods are more used than others. The iris is one of the most applied biometric patterns because it has a significant difference from person to person; does not show variations throughout the lifetime, excluding ophthalmic diseases; does not suffer wear signs and the accidents that may affect it are uncommon. Besides, it is a little invasive biometrics method $[2]$.

Depending on each region of the world, face and eye physiognomy change. An obvious example is Orientals, who have more significant iris occlusion in contrast to the Westerners who do not have such a pronounced occlusion.

D. R. Cardona-Cortes was student at the Universidad Tecnológica de Pereira, Carrera 27 \#10-02 Barrio Álamos - Risaralda - Colombia, (drcardona@utp.edu.co).

J. A. Cortés-Osorio is professor and researcher at the Universidad Tecnológica de Pereira, Carrera 27 \#10-02 Barrio Álamos - Risaralda Colombia, (jacoper@utp.edu.co). 
Nowadays, there is not a study on iris biometrics applied in Latin American eyes, because there is no public database with exclusively Latin American physiognomy.

This investigation evaluates the capability to segment and classify Latin American eyes with visible light, these ones present different characteristics of color and occlusion in relation to those ones in the data bases that are normally used in the researches about iris biometrics recognition.

This work emphasizes the iris biometric recognition by the Principal Component Analysis (PCA) method with the assistance of the Naive Bayes classifier. A database with iris images of Latin American eyes of the Colombian Andean region was created, which were processed and analyzed for individual's recognition. Finally, to test its legitimacy, the results were tested for validity.

Some studies have been made that, similarly, approach the subject of iris biometrics. Among the highlighted studies are those carried out by the ophthalmologists Flom and Safir who managed to obtain the patent of the design of an unimplemented biometric system, where they propose a controlled environment always to obtain the same pupil diameter; It indicates that the lighting must be constant to force the pupil to have a steady size. These are the first beginnings of the iris biometric recognition [1]. Subsequently, in 1994, Daugman began his research work on iris biometrics [3]. The proposed technique required the detection of the center and radius, both of the iris and the pupil, using integral-differential operators in circle arcs. He then proposed to perform a pseudo-polar transformation of the limited iris region to obtain afterward the region phase information using non-orthogonal Gabor Wavelets that were encoded later by providing a unique template called IrisCode [4]. A few years later, in 1997, Wildes introduced a system based on the Hough transform, allowing circles detection at the image contours. The eyelids were modeled by parabolic arches.

Feature extraction was based on a pyramid multi-resolution representation of iris patterns. The comparison stage used the calculation of a normalized correlation factor for each frequency band. The final decision was achieved through a linear discriminant analysis classifier [5]. The following year, in 1998, Boles and Boashash based their proposal on calculating the zero crossings of the Wavelet transform with different levels of resolution on the concentric circles of the iris, resulting in signals of a dimension that were compared with the model features using different functions of dissimilarity [6]. In 2000, Zhu, Tan, and Wang presented a recognition technique composed of an acquisition system, an image processing system, a feature extraction system, and a classifier design. The algorithm was based on textures analysis using multichannel Gabor filtering with the Wavelet transform. The authors indicated that their method was invariant to scale, rotation, and translation [7]. In 2003, Masek proposed an open-source iris recognition system. The method included a segmentation module based on the Hough transform that was used to locate the pupil, iris, eyelids, eyelashes, and reflexes.

On the other hand, the extracted regions were normalized in rectangular blocks with constant dimensions to avoid inconsistencies in the images. The phase data of the Gabor 1-D filters were extracted and quantized in four levels to encode the unique iris pattern in a biometric bit template [8]. In 2004, Rydgren et al. proposed the use of Wavelets to transform and characterize the iris texture. They also made an objective comparison with the Gabor wavelet [9]. On the other hand, Coomonte Belmonte, in 2006, proposed a system of people recognition through its iris pattern based on the Wavelet transform, which allowed the analysis of the one-dimensional signals, obtained from the iris images [10]. In 2008, Bowyer, Hollingsworth, and Flynn presented a historical development of techniques for the study of iris biometric images. They sampled research classifications and experimental evaluations about iris biometrics [11]. In 2010, Kekre, Thepade, Jain and Agrawal presented a document in which they use Haar Wavelets of various levels for iris recognition, in their results it is observed that level 5 of Haar Wavelets obtained the better result, reaching $67,5 \%$ of the true acceptance rate and $37,5 \%$ of the false acceptance rate [12]. In 2016 Luhadiya and Khedkar propose the extraction of iris characteristics using the co-occurrence technique in the grayscale. Besides, they propose to use Multiclass Vector Vector Support Machines as a classifier, obtaining 94,23\% accuracy [13].

Finally, digital image processing is not only used for biometric recognition. It has been found among national research that asphalt mixtures are analyzed from threedimensional reconstructions [14]. The analysis of digital images also contributes to forensic science, in [15] they analyze the "traces" left in a projectile when fired by a firearm to collate if they come from it.

\section{THEORICAL FRAMEWORK}

\section{A. Principal Components Analysis (PCA)}

Principal Components Analysis is a technique with a statistical approach that linearly transforms an original set of data into a smaller, uncorrelated set that represents the most significant variance of multivariate input data. This technique is widely used in face recognition because it extracts the main characteristics of the data found in the high-dimension space of the image. Its main uses are classification, reduction of a data set, feature extraction, and change detection [16].

\section{B. EigenIris}

The present research applies the procedure described in this section to use the Principal Component Analysis (PCA) technique for iris recognition. Initially, a training database with a set of $M$ images in a single color layer $I_{1}, I_{2}, \ldots I_{m}$ must be available. Subsequently, the images are normalized so that each pixel is between [ $0-255]$. Additionally, all images must have the same size $N x N^{\prime}$ to obtain a set of images $I_{1}, I_{2}, \ldots I_{m}$, where $I_{k}$ is the k-th image of size $N x N^{\prime}$ as shown in (1).

$$
I_{k}=\left[\begin{array}{cccc}
p_{1,1}^{k} & p_{1,2}^{k} & \cdots & p_{1, N^{\prime}}^{k} \\
p_{2,1}^{k} & p_{2,2}^{k} & \cdots & p_{2, N^{\prime}}^{k} \\
\vdots & \vdots & \ddots & \vdots \\
p_{N, 1}^{k} & p_{N, 2}^{k} & \cdots & p_{N, N^{\prime}}^{k}
\end{array}\right]
$$


Once this process is finished, the representation of the $I_{k}$ image of the iris must be changed from a matrix form to a vector representation as presented in (2). That is, all rows of the $I_{k}$ matrix are concatenated into a vector $\Gamma_{k}$ of dimension $\left(N \times N^{\prime}\right) \times 1$.

$$
\Gamma_{k}^{\prime}=\left[p_{1,1}^{k} \cdots p_{1, N}^{k} \cdots p_{1, N^{\prime}}^{k} \cdots p_{N, 1}^{k} \cdots p_{N, N^{\prime}}^{k}\right]
$$

Next, the average iris column vector is found among all trained irises. The average iris can be defined as $\Psi=\frac{1}{M} \sum_{i=1}^{M} \Gamma_{i}$. Following, each iris $\Gamma_{i}$ is subtracted from the average iris to obtain the characteristic vector of each iris, such as this: $\Phi_{i}=\Gamma_{i}-\Psi$.

Now, we must find the set of orthonormal vectors, see (3), that best describe the data distribution. The Eigenvalues $\lambda_{k}$ and the Eigenvectors $u_{k}$ of $C$ are used for this purpose.

$$
\begin{aligned}
C=\frac{1}{M} \sum_{i=1}^{M} \Phi_{i} \Phi_{i}^{T} & =A \cdot A^{T} \text { donde } A \\
& =\left[\Phi_{1}, \Phi_{2}, \ldots, \Phi_{M}\right]
\end{aligned}
$$

Since $A$ has dimensions $\left(N \times N^{\prime}\right) \times M$, then matrix $C$ is of dimensions $\left(N \times N^{\prime}\right) \times\left(N \times N^{\prime}\right)$ which is significantly large to manipulate. Firstly, the internal product of the matrix $L=$ $A^{T} A$, of dimensions $M \times M$, is calculated to determine the Eigenvectors and the Eigenvalues of the matrix $C$, and then the Eigenvectors $v_{i} i=1, \ldots, M$ of $L$ are found. Now, it is observed that if $L v_{i}=\lambda_{i} v_{i}$, then (4) is obtained:

$$
\begin{array}{cl}
A L v_{i} & =\lambda_{i} A v_{i} \\
A\left(A^{T} A\right) v_{i} & =\lambda_{i} A v_{i} \\
C A v_{i} & =\lambda_{i} A v_{i} \\
C u_{i} & =\lambda_{i} u_{i}
\end{array}
$$

Therefore $u_{i}=A v_{i}$ and $\lambda_{i}$ are the Eigenvectors of $M$ and the Eigenvalues of $C$, respectively. It is necessary to make sure to normalize $u_{i}$ so that $\left\|u_{i}\right\|=1$. Finally, the eigenvectors of $u_{i}$ are called Eigeniris.

The smallest values, resulting from the Eigenvectors, can be discarded as shown in (5), so $R \leq M$ values will be left, that is, the largest values, which are those that represent the largest distribution of the variance of the set of original images. Summing up, all of them are entered a matrix as depicted in (5) [17].

$$
U=\left[u_{1}, u_{2}, \ldots, u_{R}\right]_{\left(N \times N^{\prime}\right) \times R}
$$

\section{Validations of qualitive methods}

In order to validate the quantitative analysis methods used in this research, the following parameters are introduced [18]:

- True Positive are those samples that exceed the threshold level with the verification algorithm and are positive in the reference.
- $\quad$ True Negative are those samples that do not exceed the threshold level with the verification algorithm and are negative in the reference.

- False negatives correspond to those samples that do not exceed the threshold value with the verification algorithm and are positive in the reference.

- False positives: correspond to those samples that exceed the threshold value with the verification algorithm and are negative in the reference.

Two parameters, sensitivity and specificity, are related to the lower and upper limits respectively. It should be noted that both are represented as probabilities [18].

Sensitivity is defined as the capacity of the algorithm to detect positive samples when, in fact, they are.

Specificity is defined as the algorithm capacity to detect negative samples when, in fact, they are.

\section{MATERIAL AND METHODS}

A Macbook Pro15" computer with an Intel Core I7 Processor at 2,4 Ghz, $4 \mathrm{~Gb}$ of RAM, and AMD Radeon 6770M $1024 \mathrm{Mb}$ Video Card, MacOs Mavericks and MatLab 2014b was used during the development of this research work.

\section{A. Shot Process}

A supporting platform for the acquisition of the images was built, taking into account the anthropometric measurements of faces presented in [19] to ensure fixed position and the same lighting conditions during the shooting. It was set to fit the iris pictures shot at the Universidad Tecnológica de Pereira, Colombia.

The prototype built for this research, as shown in Fig. 1, had a positioning mechanism for the camera on the $\mathrm{X}, \mathrm{Y}, \mathrm{Z}$ axes so that, regardless of the size of the person's face within the regular measurements, the eye was easily located within the image's frame. Note that the previous design made in Solidworks, shown in Fig. 1, differs in some manner concerning the implemented.

Some slight differences are the chin rest and slide of the Xaxis, due to the construction difficulties with the available materials. 


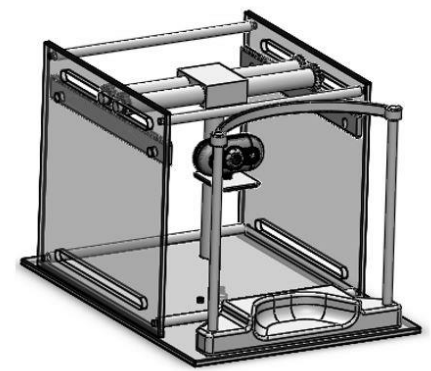

(a)

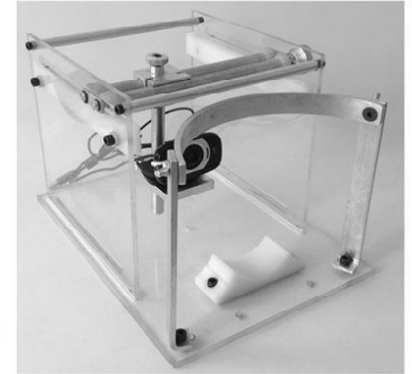

(b)
Fig. 1. (a) Prototype design made in SolidWorks ${ }^{\circledR} 2013$ and (b) finished mechanical in aluminum and acrylic. Source: Own development.

A 12 MP Logitech C615 webcam was used for the iris image capture. This camera had an electronic adjustment method that allowed to control through the computer the focal distance, to take the pictures in an eye distance range from $7 \mathrm{~cm}$ to $10 \mathrm{~cm}$.

Fig. 2 shows that there are no reflections in the iris, in addition to an image that is appropriately focused.

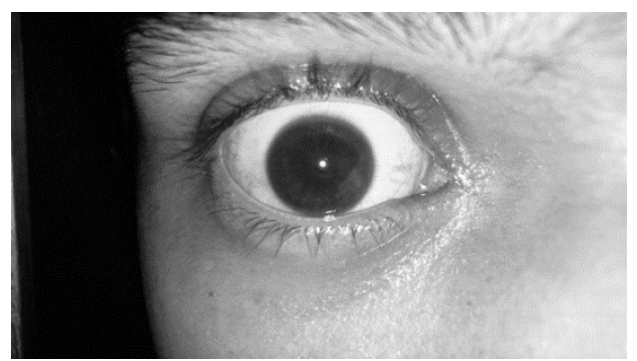

Fig. 2. Image belonging to the database, captured under constant lighting and position conditions, RGB color. Source: Own development.

The built database had a total of 500 images distributed as follows: 20 images of the right eye of 20 people, for a total of 400 (trained subjects); 5 images of the right eye of 20 people, for a total of 100 (untrained subjects). The images had a $1280 \times 720$ pixel resolution and were captured in TIFF format because it reduced artifacts produced due to poor image compression, that allowed features extraction with minimal information loss and reasonable disk storage room. Finally, the iris images were taken with the written permission of each of the participants in the database. The database is available for academic use http://academia.utp.edu.co/jacoper/biometrics/.

\section{B. Images Processing}

It was needed to apply techniques that allowed to adjust the images to obtain suitable segmentation, and thus highlight the area of interest, which was, in this case, the iris.

The incoming image was in RGB, that allowed selecting each color layer for processing. Then, each layer was extracted, and individually preprocessed to increase the contrast through the gamma adjustment.

A different Gaussian kernel was used for filtering in order to detect the iris outer edges and the pupil more accurately using the Canny method. In each case, these were 42 pixels size and
7 for the standard deviation for the iris, and 18 pixel size and 3 for the standard deviation for the pupil, respectively. See Fig. 3.

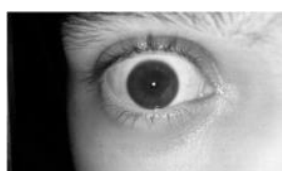

(a)

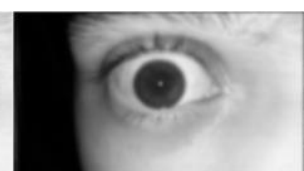

(b)

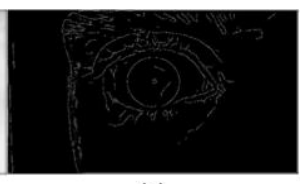

(c)
Fig. 3. (a) Red layer. (b) Image with a Gaussian kernel for smoothing with a 42 pixel side window and a standard deviation of 7 for iris extraction. (c) Image with Canny edge detector with a threshold of 0,1 for iris detection. Source: Own development.

Finally, in the image preprocessing step, a Canny edge detector was applied with a threshold of 0,1 for iris detection and 0,05 for pupil detection as seen in Fig. 3c.

\section{Iris Segmentation}

Segmentation is isolating the object of interest from the rest of the image. The circular Hough Transform allowed obtaining the coordinates of the center of the circle and its radius. This method was applied to all the binarized images after the thresholding. An example of iris location in the eye within a color image of the created database itself is presented in Fig. 4.

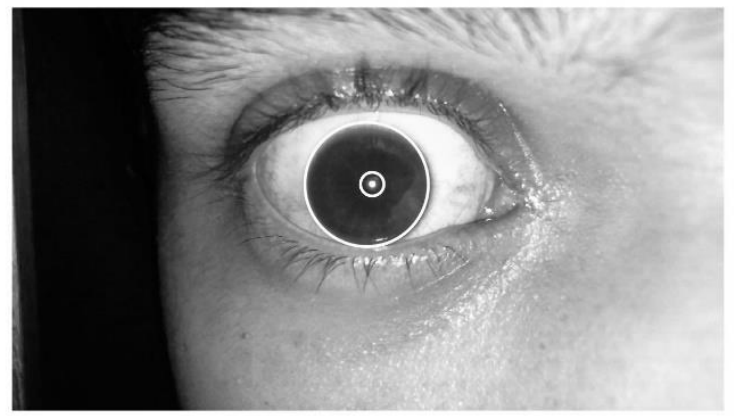

Fig. 4. Image with both the iris and pupil detected using the Hough circular transform. Source: Own development.

As noted earlier, the iris and the pupil centers and radios were obtained using the circular Hough transform to carry out the iris segmentation as shown in Fig. 5.

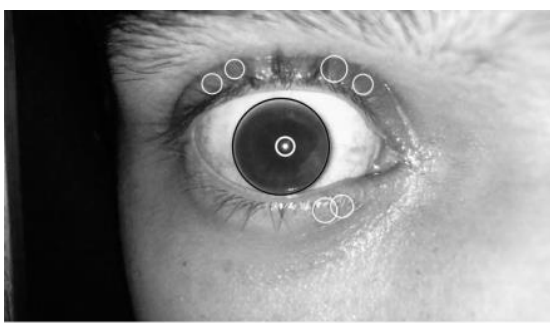

(a)

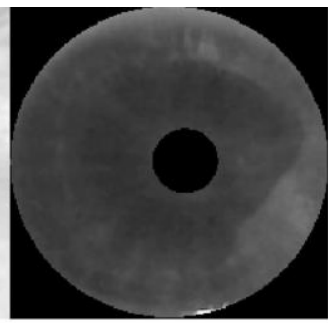

(b)
Fig. 5. (a) Image with iris and pupil detected. (b) image with the iris segmentated. Source: Own development.

Fig. 5 shows that the circular Hough transform found several circumferences while searching for the pupil. The actual center and radio of the pupil were selected the closest to the iris to solve this issue. 


\section{Standarization}

In the normalization process, the image resulting from the segmentation was transformed from a native polar to a rectangular representation using the nearest neighbor approach [6]. This process made a planar representation of the circular segmented iris image. The resulting image was 90x360 pixels resolution where 90 corresponded to the average distance value from the pupil to the iris edge and 360 to the sampling of the complete iris circumference in degrees. See Fig. 6.

At the moment of transforming to the rectangular representation, it was decided to do it in all RGB color layers in order to analyze which one was more suitable for the characteristics extraction.

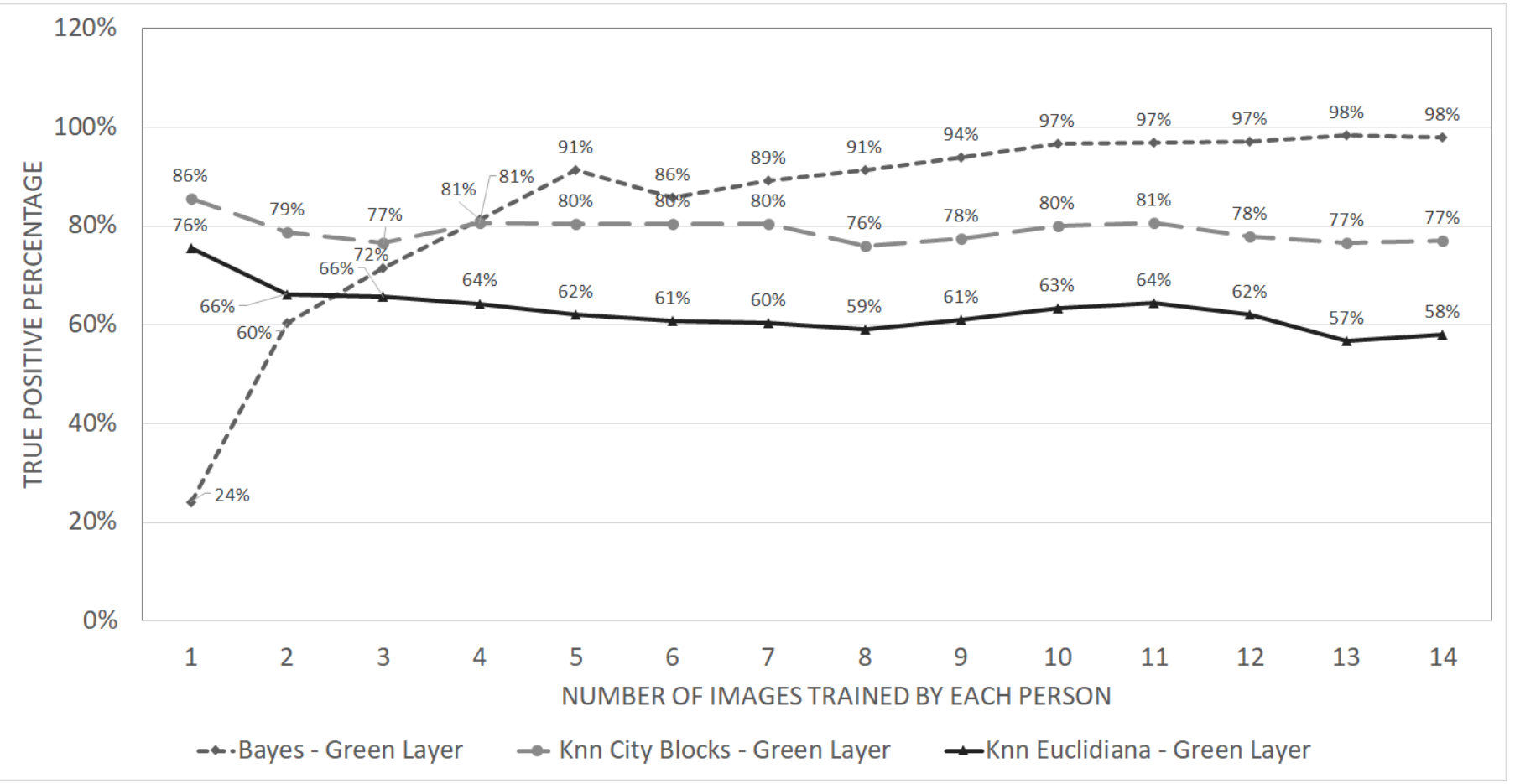

Fig. 6. Percentage graph of true positives that were obtained with the Naive Bayes and KNN classifiers with CityBlock distance and Euclidean distance for the green RGB color layer, against the number of images trained by each person. Source: Own development.
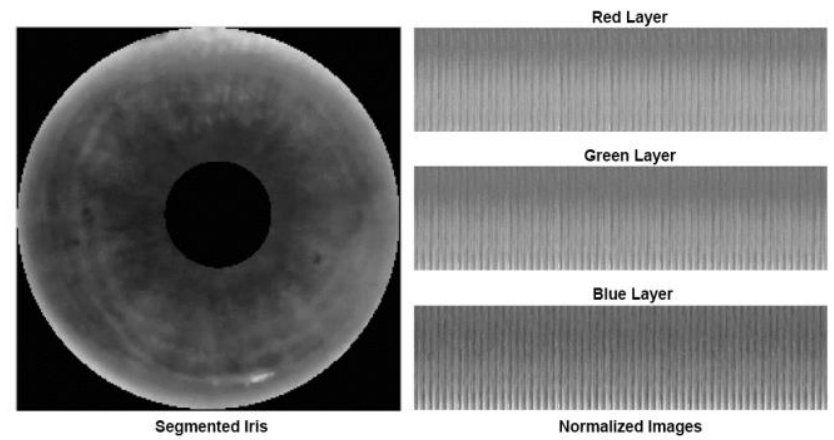

Normalized Images

Fig. 7. Left image is the segmented iris, right images are the normalized iris in each of the RGB color layers. Source: Own development.

\section{E. Training}

In this step, the Zero Average was applied to the image obtained in the normalization. This was performed to adjust all the images to the same intensity scale. Subsequently, the PCA technique was used (Eigeniris), and the characteristics were collected. The training was carried out with 15 images of 20 people's right eye for a total of 300 images.

\section{F. Recognition}

The PCA regular recognition approach was modified by substituting the basic internal distance classifier technique to the Naive Bayes classifier with a normal Gaussian distribution, and the k-nearest neighbors (KNN) classifier with Euclidean distance and Cityblock being $\mathrm{k}=1$, independently. In order to test the modified technique, 100 images of 20 trained subjects and 100 images of 20 untrained subjects, five photos of the right eye of each subject were used.

\section{G. Validity test}

Validation is a process to evaluate a proposed classification method under simulated conditions to estimate how the algorithm would run in real situations for non-trained iris data [20]. Therefore, it is crucial to validate the implemented models by comparing their performance before developing them in an application environment. In this experiment, we used holdout validation with $75 \%$ for training, $25 \%$ for testing, to estimate the false positives rate, the true positives rate, false negatives rates, the true negatives rate, the accuracy, the sensitivity, and the specificity. Additionally, the ROC Receiver Operating Characteristic curve was considered to evaluate the approaches. 


\section{RESULTS}

Different layers of RGB color were used in order to detect the iris and the pupil. We obtained $100 \%$ of accurate location in the green and red layer, both for the pupil and the iris.

Fig. 7 shows the percentage of true positives against the number of images trained by each person. The Naive Bayes classifier and KNN classifier with Euclidean distance and City
Block were evaluated. The percentages were obtained by training using the number of images mentioned in Fig. 7 and the residual amount to complete the 20 were used for recognition.

The Naive Bayes classifier had a detection threshold. Fig. 8 shows the detection threshold variation against the percentage of true positives, true negatives, false positives and false negatives.

\section{RECOGNITION THRESHOLD}

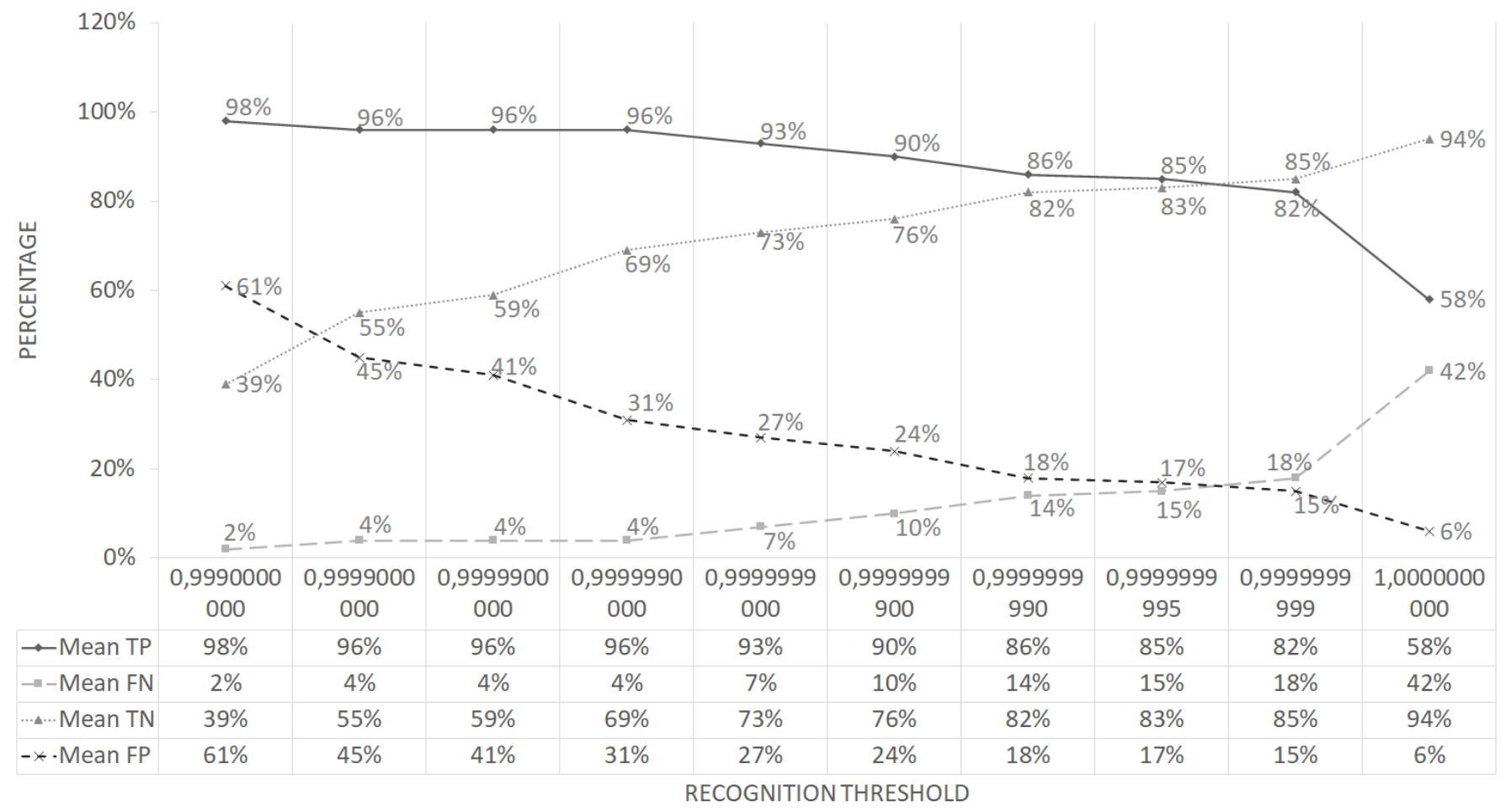

Fig. 8 Percentage graph of true positives, true negatives, false negatives and false positives using the Naive Bayes classifier with the variation of the recognition threshold. Source: Own development.

Fig. 9 shows a total of 200 samples, knowing that 100 should be true positives and 100 true negatives, however, 83 true positives, 17 false positives, 15 false negatives, and 85 true negatives were obtained. We estimated the sensitivity of $83 \%$ and the specificity of $85 \%$. The indicated results with "1" are those people who were trained, and those with "0" were not trained.

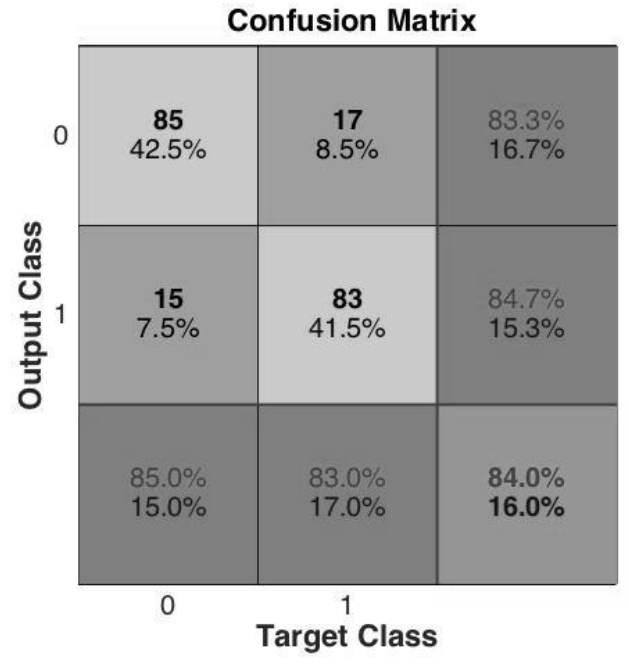

Fig. 9 Confusion matrix for a threshold of 0,99 of the binary response of the classifier as positive 
The ROC curve obtained from Fig. 9 is shown in Fig. 10, this is plotted from the obtained results using the Naive Bayes classifier in the green layer of RGB color.

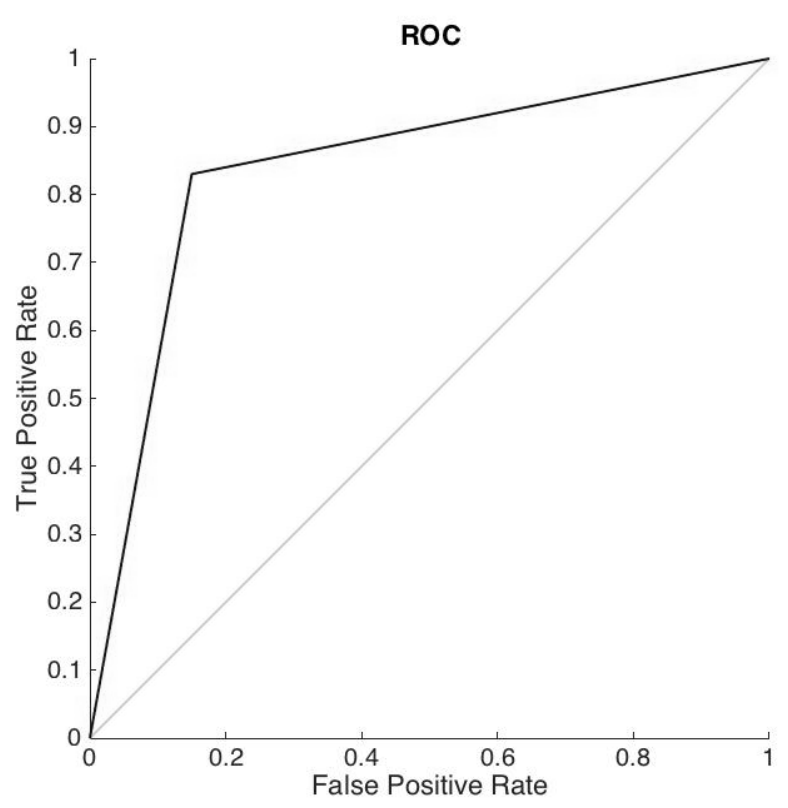

Fig. 10. ROC curve graph using Naive Bayes classifier in the green RGB color layer with a threshold of 0,99 . Source: Own development.

\section{CONCLUSIONS}

The green layer was chosen to extract the PCA characteristics, since this, in comparison with the blue layer, it reached a true positive rate of $90 \%$ with a lower number of trained images. See Fig. 7.

When comparing the trends within Fig. 7, that correspond to the evaluation of the classifier concerning the number of trained images, the use of the Naive Bayes classifier with Normal Gaussian distribution is set as the most suitable, since it has a constant growth, and from the trained image number 8 it achieves a percentage of true positives greater than $90 \%$.

Fig. 10 shows the balance between true positives, true negatives, false positives, and false negatives against a given threshold. We found that the defined threshold for the Naive Bayes classifier, with normal Gaussian cutoff distribution was 0.99 , also achieved a sensitivity of 0.82 and a specificity of 0.85 , see Fig. 1.

The authors concluded that the visible white light spectrum is also suitable for the success of biometrics and subsequent iris recognition, but at the same time, they consider that it is necessary to make a careful adjustment of the LED light intensity since most people who participated in the sample expressed discomfort to prolonged exposure to light.

\section{DISCUTION}

In [21], its authors propose to perform the segmentation firstly by locating the iris circumference and segmenting it, to later find the pupil circumference center. This way, they improved the computational cost by not scanning for the pupil center and radio in the whole image. In this work, the location of iris and pupil centers were made on the complete image, but the pupil was located efficiently by determining that it was centered within the iris, discarding the other candidates. The results obtained in the segmentation carried out in [21] were $79 \%$ for the CASIA-Iris-Interval database and for this work $100 \%$ was achieved with Latin American eyes, however, it should be taken into account that the Databases are different, with the CASIA database presenting higher iris occlusion due to Asian eye features but the images were captured with an infrared camera, allowing better iris segmentation.

In [22] the authors address the iris biometric recognition with the method based on poor representation and the PCA technique, obtaining $98,15 \%$ of recognized subjects using 6 images to train and 1 to recognize, however, in the work they do not mention whether the algorithm was subjected to tests with untrained subjects to validate their work by determining the sensitivity and the specificity against what was done in the present research. It is noteworthy that the main objective of [19] was to evaluate the robustness of the occlusion algorithm presented in the images, achieving $71,3 \%$ of subjects recognized with $30 \%$ of the iris occluded in their results.

The CASIA database is used in multiple scientific articles [23]. In this work, we used our database to evaluate the PCA technique in Latin American eyes, because the facial features and especially the eyelids and eyelashes change between races modifying the occlusion and color conditions. This is a significant contribution to the state of the art, in terms of the sample used and its additional challenges.

\section{ACKNOWLEDGMENT}

We thank those students from the Universidad Tecnológica de Pereira, who allowed us to take their iris images to carry out this research.

\section{REFERENCES}

[1] F. Serratosa, "La biometría para la identificación de las personas," Presented at Universitat Oberta de Catalunya, Barcelona, España, 2016.

[2] Iris Recognition System, by L. Flom, and A. Safir. (1987). US Patent 4641349.

[3] Biometric Personal Identification System Based on Iris Analysis, by J. Daugman. (1994). US patent 5291560.

[4] J. Daugman, "How iris recognition works," IEEE Transactions on Circuits and Systems for Video Technology, vol 14, no 1, pp. 21-30, 2004, doi: 10.1109/TCSVT.2003.818350

[5] R. Wildes, "Iris Recognition: An Emerging Biometric Technology," Proc. Of the IEEE, 1997, doi: 10.1109/5.628669

[6] W. Boles, and B. Boashash, "A human identification technique using images of the iris and wavelet transform," IEEE Transactions on Signal Processing, vol 46, no 4, pp. 1185-1188, 1998, doi: $10.1109 / 78.668573$ 
[7] Y. Zhu, T. Tan, and Y. Wang, "Biometric Personal Identification Based on Iris Patterns," Presented at 15th International Conference on Pattern Recognition, 2000, doi: 10.1109/ICPR.2000.906197

[8] L.Masek, "Recognition of Human Iris Patterns for Biometric Identification," School of Computer Science and Software Engineering, Universidad de Western Australia, Perth, 2003.

[9] E. Rydgren, E. Thomas, F. Amiel, F. Rossant, and A. Amara, "Iris features extraction using wavelet packets," Presented at International Conference Image Processing, Singapore, Singapore, 2004, doi: 10.1109/ICIP.2004.1419435

[10] R. Coomonte, "Sistema de reconocimiento de personas mediante su patrón de Iris Basado en la Transformada Wavelet," Escuela Técnica Superior de Ingenieros de Telecomunicaciones, Madrid, 2006.

[11] K. Bowyer, K. Hollingsworth, and P. Flynn, "Image Understanding for Iris Biometrics: A Survey," Computer Vision and Image Understanding, vol 110, no 2, pp. 281-307, 2006, doi: 10.1016/j.cviu.2007.08.005

[12] D. Kekre, S. Thepade, J. Jain, and N. Agrawal, "IRIS Recognition using Texture Features Extracted from Haarlet Pyramid," International Journal of Computer Applications, vol 11, no 12, pp. 1-5, 2010, doi: $10.1145 / 1980022.1980038$

[13] R. Luhadiya, and A. Khedkar, "Iris detection for Person Identification using Multiclass SVM," Presented at IEEE International Conference on Advances in Electronics. Pune, India, 2016, doi: 10.1109/ICAECCT.2016.7942619

[14] W. D. Fernández, J. D. Pacateque, M. S. Puerto, M. I. Balaguera, and F. Reyes, "Asphalt mixture digital reconstruction based on CT images," Ciencia e Ingeniería Neogranadina, vol 25, no 1, pp. 17 - 25, 2015.

[15] J. Mendez, J. H. Rivera, and J. A. Soto, "Reconocimiento de texturas en imágenes de proyectiles: un aporte a la identificación automática de armas," Ciencia e Ingeniería Neogranadina, vol 22, no1, pp. 123 - 137, 2012.

[16] M. Richardson, "Principal Component Analysis," University of Oxford, 2009 [Online] Available: http://www.sdss.jhu.edu/ szalay/class/2015/SignalProcPCA.pdf.

[17] M. Zabarauskas, "Eigenfaces Tutorial," 2009 [Online] Available: http://blog.zabarauskas.com/eigenfaces-tutorial/.

[18] D. Massart, "Handbook of chemometrics and qualimetrics," Elsevier Science, ed 3rd, 1997, pp. 886.

[19] Clínica Dr Arquero. "Las medidas de la nariz, la cara y la cabeza," 2013, [Online] Available: http://www.rinoplastia.eu/106_medidas.html.

[20] Y. Liu, "Python Machine Learning by Example easy-to-follow examples that get you up and running with machine learning," Birmingham Mumbai: Packt Publishing, 2017.

[21] J. Valencia, J. Cruz, L. Caicedo, and C. Chamorro, "Extracción de características del iris como mecanismo de identificación biométrica,". Revista Virtual Universidad Católica del Norte, vol 12, no 42, pp. 182196, 2014, [Online] Available: http://revistavirtual.ucn.edu.co/index.php/RevistaUCN/article/view/5 03/1042

[22] Z. Yang, and C. Qing, "A new iris recognition method based on PCA and sparse representation towards occlusion," Presented at IEEE International Conference Consumer Electronics. China, 2014 doi: 10.1109/ICCE-China.2014.7029879

[23] CASIA Iris Image Database, Center for Biometrics and Security Research. 2002, [Online] Available: http://www.cbsr.ia.ac.cn/IrisDatabase.htm.

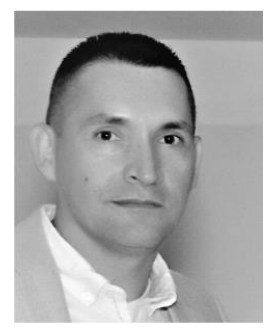

Jimy Alexander Cortés-Osorio was born in Pereira, Colombia, in 1968. He received the B.E. degree in electrical engineering and the M.S. degree in physics instrumentation from the Universidad Tecnológica de Pereira (UTP), Pereira, in 1999 and 2009, respectively. He also completed his Ph.D. degree in engineering with the
Universidad Nacional de Colombia (UNAL), Colombia in 2020. From 2003 to 2012, he was a Lecturer with the Department of Physics, UTP, where he has been a Full Professor since 2013. His current research interests include image processing, image motion analysis, machine vision, machine learning, and instrumentation and measurement. Mr. Cortés-Osorio is an Affiliate Member of the Society for Industrial and Applied Mathematics (SIAM) and the Institute of Electrical and Electronics Engineers (IEEE).

ORCID: https://orcid.org/0000-0002-0413-807X

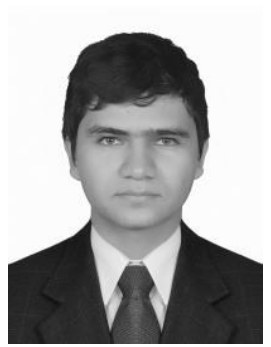

Deivy Alejandro Muñoz-Acosta was born in La Tebaida, Quindio, Colombia in 1993. He received the B.E degree in Mechatronic Ingineering and the M.S. degree in Physics Instrumentation from Universidad Tecnológica de Pereira (UTP) in 2015 and 2018, respectively. He works for Department of Technologies as a professor since 2015 . His currently research include image processing and machine learning.

ORCID: https://orcid.org/0000-0003-4743-8301

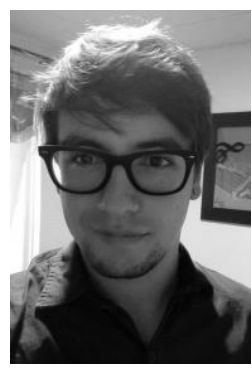

Daniel Ricardo Cardona Cortes, He was born in Manizales, Colombia. $\mathrm{He}$ is a Mechatronic Engineer from Universidad Tecnologica de Pereira, Colombia. He worked as a teacher at Colegio los Andes in Chinchiná for 1 year. Later, he held the position of Mechatronic in the company Ingeniería AudioVisual S.A.S. Currently, he owns the company AutoCal Instrumentación S.A.S, where he performs calibration of Metrology equipment.

ORCID: https://orcid.org/0000-0002-5851-2143 